\title{
Granuloma Periférico de Células Gigantes e Hiperparatiroidismo. Presentación de un Caso
}

\author{
Peripheral Giant Cell Granuloma and Hyperparathyroidism. Case Report
}

\author{
Ana Alarcón Arratia ${ }^{1,2}$; Maximiliano Muñoz Repetto3; Katty Schnettler ${ }^{4}$ \& Carolina Ulloa Marin ${ }^{5}$
}

ALARCÓN, A.; MUÑOZ, R. M.; SChNeTTLER, K. \& ULLOA, M. C. Granuloma periférico de células gigantes e hiperparatiroidismo. presentación de un caso. Int. J. Odontostomat., 13(3):266-270, 2019.

RESUMEN: Se presenta el caso de un paciente sexo femenino 31 años, insuficienciente renal crónica en hemodiálisis, hipertensa secundaria, consulta por aumento de volumen oral en relación a encía marginal vestibular izquierda, 6 meses de evolución. Al examen se observa aumento de volumen de $15 \mathrm{~mm}$ en relación a piezas. 3.6 y 3.7. Radiografía muestra compromiso periodontal pieza 3.7. Se realiza biopsia excisional y exodoncia pieza 3.7. Histopatología informa granuloma periférico de células gigantes. Se solicitan exámenes de laboratorio para estudio de hiperparatiroidismo (PTH elevada, calcemia fosfatemia normales). Evoluciona favorablemente. Sin signos de recidiva a los 3 meses. El diagnóstico histopatológico de lesión de células gigantes debe ser complementado con la clínica, exámenes de laboratorio e imagenología, el objetivo final es definir si tal diagnóstico corresponde a un tumor pardo, a un granuloma central de células gigantes o a un granuloma periférico de células gigantes. En la literatura los reportes de asociación entre granuloma periférico de células gigantes e hiperparatiroidismo son escasos. Este caso corresponde a granuloma periférico células gigantes, asociado a hipersecreción de paratohormona.

PALABRAS CLAVE: granuloma periférico, células gigantes. hiperparatiroidismo. insuficiencia renal crónica.

\section{INTRODUCCIÓN}

Lesiones de células gigantes (tumor pardo, granuloma central de células gigantes y en menor frecuencia granuloma periférico de células gigantes) pueden presentarse en los huesos o gingiva de un paciente con hiperparatiroidismo secundario a una insuficiencia renal crónica (IRC) (Triantafillidou et al., 2006; Araya et al., 2014). Estas lesiones presentan una histopatología prácticamente idéntica (Triantafillidou et al.; Araya et al.; Vaidya et al., 2018), constituida por células gigantes multinucleadas, células fibrohistiocíticas mononucleares, fibroblastos y eritrocitos extravasados. El desafío frente al dignóstico histopatológico de lesión de células gigantes en un paciente con IRC se encuentra en lograr una adecuada anamnesis, examen físico, exámenes de laboratorio e imágenes que aporten datos esenciales a la hora de proponer uno $u$ otro diagnóstico, puesto que las alternativas terapéuticas varían considerablemente según sea el caso.
La IRC es la persistencia de anormalidades estructurales o funcionales del riñón por tres o más meses. Es producto del daño renal debido a cambios estructurales del riñón y/o del tracto urinario ocasionando una alteración en la composición de la orina, reducción de la función renal o ambas. Su prevalencia ha ido en aumento siendo reconocido como un problema de salud a nivel mundial, llegando a tener valores estimados de un $10 \%$ de la población mundial (Zhang et al., 2012). Su tratamiento termina siendo la hemodiálisis y finalmente el trasplante renal. En el transcurso de la IRC se producen cambios homeostáticos que derivan en aparición de enfermedades secundarias como lo son la hipertensión arterial e hiperparatiroidismo. El hiperparatiroidismo primario se caracteriza por hipersecreción de PTH, hipercalemia e hipofosfatemia y por lo general es producto de un adenoma en la glándula paratiroídea (85\% de los casos). El hiperparatiroidismo secundario se caracteriza por

\footnotetext{
${ }^{1}$ Servicio de Cirugía Oral y Maxilofacial, Hospital Clínico San Borja Arriarán. Santiago, Chile.

${ }^{2}$ Departamento de Cirugía Oral y Máxilofacial. Facultad de Odontología, Universidad de Chile, Santiago, Chile.

${ }^{3}$ Cirujano Dentista, práctica privada.

${ }^{4}$ Servicio de Anatomía Patológica, Hospital Clínico San Borja Arriarán,Santiago, Chile.

${ }^{5}$ Servicio Cirugía Maxilofacial, Hospital de la Unión, La Unión, Chile.
} 
hipersecresión de PTH, hipocalcemia e hiperfosfatemia (Triantafillidou et al.; Araya et al.). En todos los pacientes con IRC que reciben diálisis debemos sospechar de un posible hiperparatiroidismo (Abou-Elfadl et al., 2014). Al respecto debemos considerar que la lesión de los maxilares que más frecuentemente se asocia a pacientes con hiperparatiroidismo es el tumor pardo.

El tumor pardo es una lesión osteolítica benigna localmente agresiva, secundaria a una alteración metabólica. Puede localizarse en cualquier hueso de la economía, siendo más frecuente en tibia, fémur, clavícula, costillas y pelvis e infrecuentemente afecta huesos faciales (2\%). Su crecimiento es lento, pudiendo presentarse de forma asintomática o bien sintomática cuando la lesión es de mayor extensión. Además puede comprometer estructuras vecinas tales como dientes y en casos extremos ocasionar deformidades faciales y fracturas patológicas (Triantafillidou et al.; Araya et al.). Al examen clínico puede ser pesquisable cuando expande y/o perfora corticales llegando a comprometer la mucosa oral. Afecta en mayor proporción a mujeres entre la 4ta y 5 ta década de vida. Radiográficamente el tumor pardo se aprecia como una o varias lesiones osteolíticas, sin esclerosis periférica, de tamaño variable uni o multiloculadas. El tratamiento en base al control médico del hiperparatiroidismo, logra una rápida remisión de la lesión, que en algunos casos puede ser parcial, realizando en una segunda etapa la extirpación quirúrgica de la lesión con curetaje (Triantafillidou et al.; Wilson et al., 2013).

Por otro lado el granuloma periférico de células gigantes (GPCG) es una lesión reaccional exofítica benigna que se origina a partir del tejido conectivo del periostio o de la membrana del ligamento periodontal localizado en encía y hueso alveolar. Se asocia a factores irritantes locales como depósitos duros, impacto alimenticio, restauraciones desajustadas, etc. Tiene una ligera inclinación por el sexo femenino, mostrando una mayor incidencia en la 4ta década de vida. Algunos autores describen su asociación a condiciones sistémicas como el hiperparatiroidismo (Smith et al., 1988; Guimarães et al., 2006; Choi et al., 2008; Boffano et al., 2013; Patil et al., 2014), siendo menos frecuente esta asociación que a las otras lesiones de células gigantes como el tumor pardo y el granuloma central de células gigantes. Clínicamente se observa como una masa pediculada, bien delimitada de color rojo oscuro y que puede sangrar a la palpación. Puede dejar una impronta en el tejido óseo subyacente, pero no debiera ocasionar destrucción ósea. El tratamiento consiste en la extirpación quirúrgica de la lesión con legrado del hueso y periostio además de eliminar el factor irritante local (Smith et al.; Choi et al.; Boffano et al.), logrando bajos niveles de recurrencia 5 - 17,5\% (Boffano et al.; Patil et al.; Lester et al., 2014).

Por su parte el granuloma central de células gigantes (GCGG) es una lesión intraósea benigna que se divide en 2 categorías: a) Lesiones no agresivas: es la más frecuente, usualmente asintomática, de crecimiento lento y sin perforación de corticales o reabsorción radicular de los dientes incluidos en la lesión y b) Lesiones agresivas caracterizadas por dolor, crecimiento rápido, exudado, parestesia, reabsorción radicular y perforación de cortical ósea comunicándose con la cavidad oral teniendo un mayor porcentaje de recurrencias comparando a la forma no agresiva. Radiográficamente se ve como una lesión radiolúcida no corticalizada, bien delimitada, uni o multilocular. El tratamiento depende del tamaño y agresividad de la lesión, normalmente se trata mediante curetaje extenso pero frente a lesiones agresivas y/o de gran tamaño se pueden realizar infiltraciones de corticoesteroides, calcitonina, triamcinolona e interferón alfa-2a de manera seriada para disminuir el tamaño de la lesión antes de realizar una cirugía más radical. Las formas agresivas muestran una alta tasa de recurrencia (11-50 \%) (Waldron, 2008; Valentine \& Nelson, 2011). Al igual que el tumor pardo, el GCCG se encuentra asociado al hiperparatiroidismo (Suárez-Roa et al., 2009; Patil et al.).

En este reporte de caso clínico, buscamos destacar la importancia de complementar el diagnóstico histopatológico de lesiones de células gigantes con exámenes de laboratorio, imágenes y examen clínico para poder así determinar el diagnóstico diferencial entre las distintas lesiones de células gigantes.

\section{PRESENTACIÓN DEL CASO}

Paciente sexo femenino, 31 años de edad acude a la unidad de cirugía maxilofacial por aumento de volumen intraoral en relación a encía marginal vestibular del lado izquierdo, de 6 meses de evolución, insuficiencia renal crónica en hemodiálisis 3 veces por semana hace 2 años, hipertensión arterial secundaria, sin otros antecedentes. Al examen se observa aumento de volumen de $15 \mathrm{~mm}$ de diámetro mayor en relación a encía vestibular entre piezas 3.6 y 3.7, de consistencia firme, base sésil, eritematoso, ligeramente doloroso a la palpación y que aparentemente desplaza pieza 3.7 la que a su vez presenta 
movilidad grado II. Presenta depósitos duros moderados y ausencia de caries (Fig. 1).

La radiografía panorámica (Fig. 2) evidencia compromiso periodontal entre piezas 3.6 y 3.7 .

La paciente se había realizado previamente una biopsia incisional de la zona, el diagnóstico describió hallazgos compatibles con granuloma periférico de células gigantes, tras lo cual la lesión comenzó a crecer aceleradamente por lo que se planifica la exéresis completa previa interconsulta a periodoncia. Se solicitaron además los siguientes exámenes de laboratorio: Hormona paratiroidea (PTH) fosfatemia y calcemia. El nivel de PTH se encontró elevado a $871 \mathrm{pg} / \mathrm{ml}$ (normal $12-72 \mathrm{pg} / \mathrm{ml}$ ), calcemia $8,9 \mathrm{mg} / \mathrm{dl}$ (normal $8,1-10,5 \mathrm{mg} /$ dl) y fosfatemia $3,2 \mathrm{mg} / \mathrm{dl}$ (normal $2,5-5 \mathrm{mg} / \mathrm{dl}$ ), hallazgos compatibles con hiperparatiroidismo secundario a IRC terminal.

Bajo anestesia local se realiza la exéresis completa de la lesión y exodoncia de pieza 3.7 debido al compromiso periodontal y pobre pronóstico que presen-

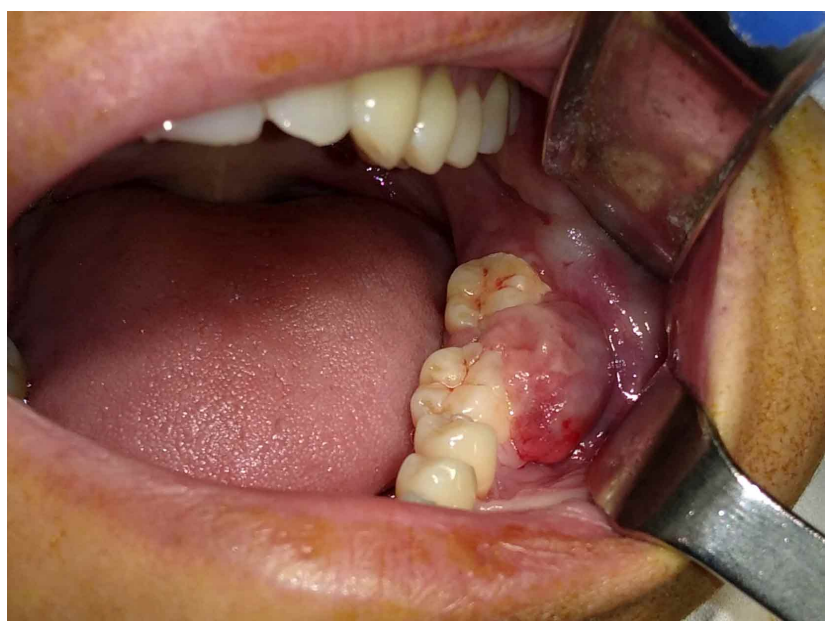

Fig. 1. Vista preoperatoria de la lesión.

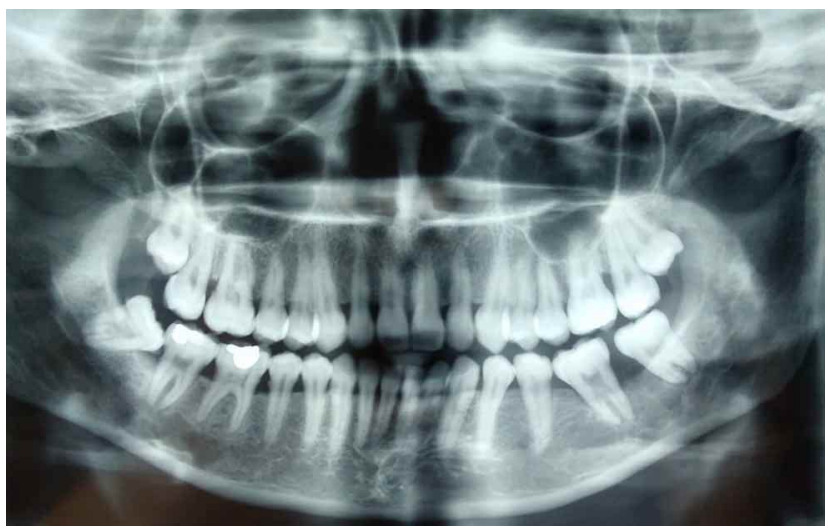

Fig. 2. Radiografía panorámica. taba. El tejido óseo no se encontraba afectado (Fig. 3). El informe histopatológico de la pieza quirúrgica nuevamente describe proliferación fusocelular entremezclada con células gigantes multinucleadas, compatible GPCG (Fig. 4).

La paciente evolucionó favorablemente sin signos de recidiva de la lesión a los 3 meses (Fig. 5).

Se informó a la paciente acerca del resultado de PTH alterada y se derivó a su médico tratante.

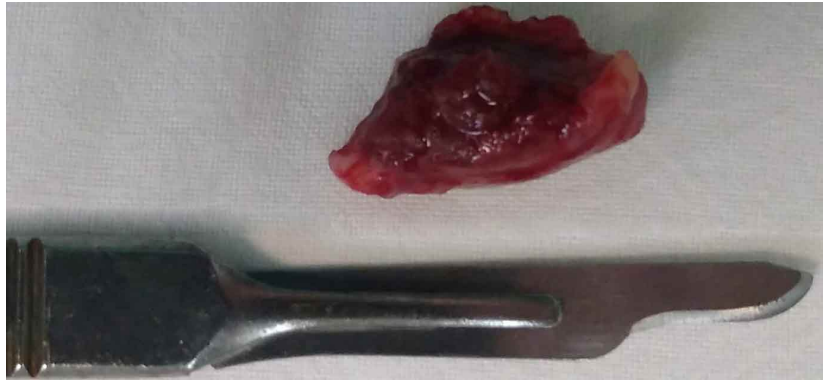

Fig. 3. Muestra excisional.

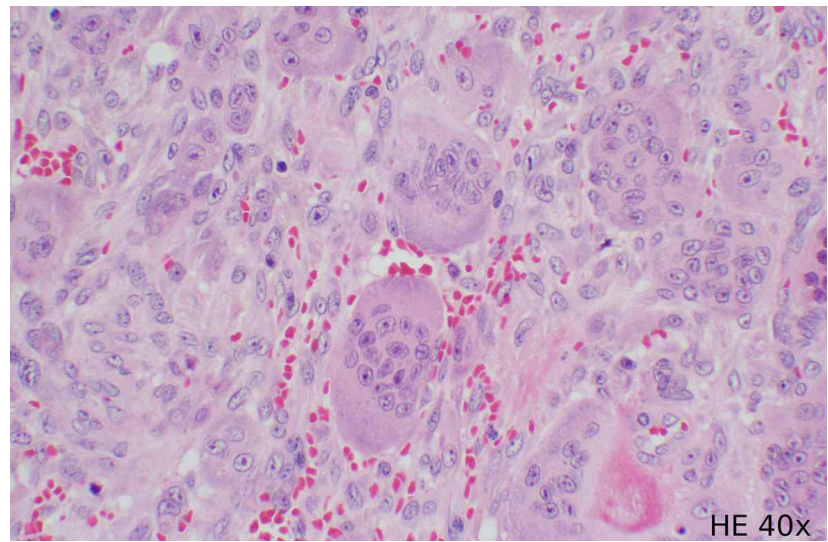

Fig. 4. Imagen de corte histológico.

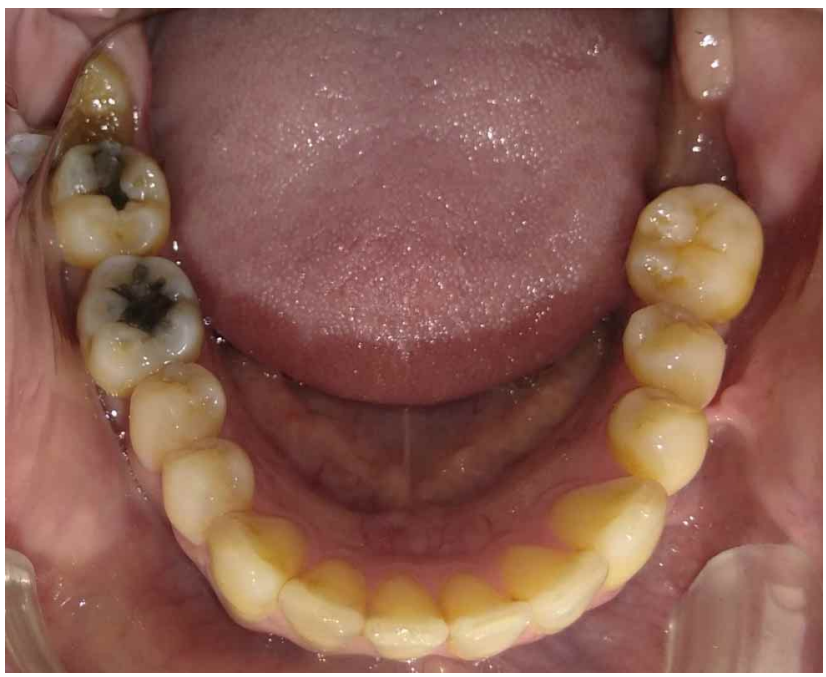

Fig. 5. Control a los 3 meses. 


\section{DISCUSIÓN}

El diagnóstico de lesiones de células gigantes no debe ser realizado sólo en base a la histología, sino que debe ser complementado con la clínica, exámenes de laboratorios e imagenología. En este caso la lesión de células gigantes corresponde a un GPCG, asociado a hipersecreción de paratohormona. Si bien sabemos que el diagnóstico más frecuente en pacientes con hiperparatiroidismo (PTH aumentada, hipocalcemia, hiperfosfatemia) es el tumor pardo, en este caso la paciente mostraba sólo alteraciones en los niveles de PTH sin alteraciones actuales en los exámenes de calcemia y fosfatemia, además en la imagen radiográfica no se pesquisó un área radiolúcida central como es característico del tumor pardo, sino que más bien correspondía a una lesión periférica, lo cual fue corroborado al momento de realizar la cirugía. Se solicitó una tomografía axial computarizada para descartar cualquier otra lesión ósea de los maxilares que no fuera identificable en la radiografía panorámica, desechando la posibilidad de que lalesión periférica sea una exteriorización de una lesión central. Finalmente todos los hallazgos apoyaron el diagnóstico de GPCG.

Por lo general el diagnóstico de GPCG no genera sospechas de hiperparatiroidismo, pero en el caso presentado se tiene el antecedente de una paciente con IRC por lo que se solicitan los exámenes de laboratorio pertinentes encontrando alterado los niveles de PTH.

Son escasos los reportes en la literatura de asociación u coincidencia de GPCG e hiperparatiroidismo (Smith et al.; Guimarães et al.; Choi et al.; Patil et al.) en donde se observa la lesión manteniendo indemnidad ósea, por lo que nos parece de especial interés el caso presentado. En un estudio realizado en Italia en un universo de 874 pacientes con diagnóstico de GPCG, 28 tenían hiperparatiroidismo (Boffano et al.), en otro estudio en Estados Unidos, de una serie de 242 pacientes con diagnóstico de GPCG se encontró a 4 pacientes que tenían hiperparatiroidismo (Lester et al.) asímismo, en otro estudio realizado por Patil et al., el hiperparatiroidismo debe ser considerado como un factor etiológico de GPCG en casos de lesiones múltiples o recurrencia de éstas, considerando que una producción desregulada de PTH puede dar origen a lesiones de células gigantes (Abou-Elfadl et al.; Patil et al.). La PTH es la hormona responsable de mantener un equilibrio entre los niveles de calcio y fósforo en el medio extracelular (Smith et al.), un incremento de la PTH puede dar origen a lesiones osteolíticas en los huesos, siendo infrecuente la localización en los maxilares (Triantafillidou et al.; Araya et al.; Abou-Elfadl et al.; Wilson et al.).

En la literatura se define que las lesiones periféricas de células gigantes pueden ser exteriorizaciones de lesiones intraóseas, por lo que es necesaria una adecuada exploración tanto clínica como imagenológica (Choi et al.; Waldron). El hiperparatirodismo generalmente es diagnosticado mediante exámenes sanguíneos de rutina, previo a la aparición de lesiones óseas. Menos del $5 \%$ de los casos identificados se manifiestan con compromiso óseo como primer signo de sospecha de hiperparatiroidismo. La presencia de este tipo de lesiones podría llegar a ser la primera manifestación de un hiperparatiroidismo primario o secundario no diagnosticado (Smith et al.; Triantafillidou et al.; Abou-Elfadl et al.; Wilson et al.).

De todos modos creemos que ante este tipo de lesiones no está demás realizar el diagnóstico diferencial con tumor pardo debido a hiperparatiroidismo.

Al pesquisar alteraciones evidentes en los exámenes de laboratorio, es necesario también esclarecer la causa de tales alteraciones. En nuestro caso la paciente padece de IRC, que puede explicar un hiperparatiroidismo secundario (PTH aumentada); sin embargo, la misma situación puede aparecer en un paciente aparentemente "sano". Lo recomendado, en ese caso sería solicitar interconsulta a endocrinología con hemograma completo y función renal además de una ecografía de cuello para descartar hiperplasias de tejido paratiroídeo o adenomas paratiroídeos que pudiesen explicar el hiperparatiroidismo.

\section{CONCLUSIÓN}

El hiperparatiroidismo puede ser un factor etiológico en la persistencia y/o recurrencia de este tipo de lesiones por lo que es de gran importancia el manejo sistémico de esta patología por parte del equipo médico para mejorar el pronóstico y disminuir posibilidad de recidiva. Finalmente, también se debe mencionar que existe la posibilidad de que el hiperparatiroidismo y el GPCG sean dos eventos independientes por lo que es necesario mayor cantidad de estudios para poder comprobar esta asociación. 
ALARCÓN, A.; MUÑOZ, R. M.; SCHNETTLER, K. \& ULLOA, M. C. Peripheral giant cell granuloma and hyperparathyroidism. Case report. Int. J. Odontostomat., 13(3):266-270, 2019.

ABSTRACT: A 31-year-old female patient with chronic renal insufficiency in hemodialysis and secondary hypertension consulted due to an enlarging intraoral lesion in relation to the left marginal vestibular gingival, with 6 months of evolution. A $15 \mathrm{~mm}$ mass in relation to mandibular left molars was noted. Radiograph showed periodontal involvement of mandibular left second molar. Excisional biopsy and tooth 3.7 extraction were performed. Histopathology reported a peripheral giant cell granuloma. Laboratory tests were requested for suspected hyperparathyroidism (aiding in the diagnosis) (high PTH, normal phosphatemia and calcemia). Evolution was favorable, without signs of reappearance after 3 months. The histopathological diagnosis of giant cell lesions should be complemented with clinical, laboratory and imaging tests. The final objective is to make the differential diagnosis between brown tumor, central giant cell granuloma and peripheral giant cell granuloma because the treatment varies considerably. There are few reports in the literature about the association between peripheral giant cell granuloma and hyperparathyroidism. This case corresponds to peripheral giant cell granuloma, possibly associated with parathormone hypersecretion.

KEY WORDS: granuloma, giant cell, hyperparathyroidism, secondary. chronic kidney failure.

\section{REFERENCIAS BIBLIOGRÁFICAS}

Abou-Elfadl, M.; Belamkaddem, A.; Mahtar, M.; Roubal, M. \& Kadiri, F. Maxillary tumor revealing primary hyperparathyroidism. Rev. Stomatol. Chir. Maxillofac. Chir. Orale, 115(5):323-6, 2014.

Araya, I.; Yanine, N.; Cornejo, M. \& Villanueva, J. Tumor pardo maxilar refractario a tratamiento convencional. Rev. Esp. Cir. Oral Maxilofac., 36(1):21-6, 2014

Boffano, P.; Benech, R.; Roccia, F.; Gallesio, C.; Garzaro, M. \& Pecorari, G. Review of peripheral giant cell granulomas. J. Craniofac. Surg., 24(6):2206-8, 2013.

Choi, C.; Terzian, E.; Schneider, R. \& Trochesset, D. A. Peripheral giant cell granuloma associated with hyperparathyroidism secondary to end-stage renal disease: a case report. J. Oral Maxillofac. Surg., 66(5):1063-6, 2008.

Guimarães, A. L. S.; Marques-Silva, L.; Gomes, C. C.; Castro, W. H.; Mesquita, R. A. \& Gomez, R. S. Peripheral brown tumour of hyperparathyroidism in the oral cavity. Oral Oncol. Extra, 42(3):91-3, 2006.

Lester, S. R.; Cordell, K. G.; Rosebush, M. S.; Palaiologou, A. A. \& Maney, P. Peripheral giant cell granulomas: a series of 279 cases. Oral Surg. Oral Med. Oral Pathol. Oral Radiol., 118(4):47582, 2014.

Patil, K. P.; Kalele, K. P. \& Kanakdande, V. D. Peripheral giant cell granuloma: A comprehensive review of an ambiguous lesion. J. Int. Clin. Dent. Res. Organ., 6(2):118-25, 2014.

Smith, B. R.; Fowler, C. B. \& Svane, T. J. Primary hyperparathyroidism presenting as a "peripheral" giant cell granuloma. J. Oral Maxillofac. Surg., 46(1):65-9, 1988.

Suárez-Roa, M. de L.; Reveiz, L.; Ruíz-Godoy Rivera, L. M.; AsbunBojalil, J.; Dávila-Serapio, J. E.; Menjívar-Rubio, A. H. \& MenesesGarcía, A. Interventions for central giant cell granuloma (CGCG) of the jaws. Cochrane Database Syst. Rev., 7(4):CD007404, 2009.

Triantafillidou, K.; Zouloumis, L.; Karakinaris, G.; Kalimeras, E. \& lordanidis, F. Brown tumors of the jaws associated with primary or secondary hyperparathyroidism. A clinical study and review of the literature. Am. J. Otolaryngol., 27(4):281-6, 2006.

Vaidya, K. ; Sarode, G. S.; Sarode, S. C.; Majumdar, B. \& Patil, S. Peripheral giant cell granuloma recurring as an exclusively intraosseous lesion: An unusual clinical presentation. Clin. Pract., 8(1):1023, 2018.

Valentine, J. C. \& Nelson, B. L. Central giant cell lesion. Head Neck Pathol., 5(4):385-8, 2011.

Waldron, C. A. Bone Pathology. En: Neville, B.; Damm, D.; Allen, C. \& Bouquot, J. Oral and Maxillofacial Pathology. $3^{\text {rd }}$ ed. St. Louis, Saunders, 2008.

Wilson, J. J.; Schwartz, H. C. \& Tehrany, G. M. Brown tumor of the posterior maxilla as initial manifestation of primary hyperparathyroidism: case report. J. Oral Maxillofac. Surg., 71(5):886-90, 2013.

Zhang, L.; Wang, F.; Wang, L.; Wang, W.; Liu, B.; Liu, J.; Chen, M.; $\mathrm{He}, \mathrm{Q} . ;$ Liao, Y.; Yu, X.; et al. Prevalence of chronic kidney disease in China: a cross-sectional survey. Lancet, 379(9818):815-22, 2012.

Dirección para correspondencia:

Ana Alarcón Arratia

Facultad de Odontología, Universidad de Chile

Sergio Livingstone Pohlhammer 943

Independencia

Santiago

CHILE

Email: anaalarcarr@gmail.com

Received: 07-07-2018

Accepted: 08-04-2019 